\title{
L'apprentissage par projet en électronique numérique
}

\author{
Wilfried Uhring ${ }^{\mathrm{a}}$, Vincent Frick ${ }^{\mathrm{a}}$ \\ a IUT de Haguenau, Université de Strasbourg, Strasbourg, France \\ Contact email : nom.prenom@unistra.fr
}

Ce papier relate une expérience d'apprentissage par projet dans le cadre du module d'électronique numérique de première année à l'IUT d'Haguenau. L'apprentissage par projet met l'apprenant au cœur de son processus de formation par le biais d'une mission à accomplir. Il identifie lui-même ce qu'il doit apprendre pour pouvoir mener à bien cette mission en jouant le rôle d'animateur, de scribe, de secrétaire ou bien d'intendant au sein d'un groupe de 4 étudiants. L'apprentissage passe donc essentiellement par un travail personnel. Un exemple de déroulement pratique ainsi qu'une mission type sont décrits dans l'article. Le retour d'expérience indique que l'approche est globalement bien perçue par les apprenants, mais elle montre des limites liées à la motivation et la maturité du public visé.

\section{Introduction}

Le programme pédagogique national (PPN) du diplôme universitaire de technologie (DUT) en génie électrique et informatique industrielle (GEII) spécifie un module d'enseignement des systèmes d'information numérique (SIN). Programmé lors du premier semestre, ce module porte sur les concepts de l'électronique numérique, de l'algèbre de Boole aux machines à état décrite en langage de description matériel. Les promotions d'étudiants accueillies au département GEII de l'IUT d'Haguenau sont majoritairement constituées de bachelier S et STI2D. Les résultats universitaires obtenus à l'IUT par ces bacheliers sont très inégaux. En effet, on constate que globalement, les bacheliers $\mathrm{S}$ se retrouvent en tête et les STI2D en queue de promotion. Cette tendance s'observe également dans le module de SIN. Ce fait peu paraitre surprenant, car parmi les étudiants de STI2D, beaucoup ont suivi l'option SIN durant leurs années de lycée alors que les étudiants de bas $\mathrm{S}$ avec option SVT découvrent cette matière à leur arrivée à l'IUT. Il semblerait donc qu'une initiation à l'électronique numérique pré-bac n'impacte pas significativement les résultats obtenus post-bac. En effet, les prérequis pour la compréhension des concepts utilisés en électronique numérique de niveau GEII $1^{\text {ère }}$ année ne sont pas très complexes et s'acquièrent rapidement. Nous avons constaté que l'implication des étudiants est le facteur principal pour la réussite universitaire et que par conséquent, c'est le travail personnel qui fait la différence. En effet, avec un travail personnel suffisant, les bacheliers de la série $S$ avec option SVT obtiennent des résultats souvent bien meilleurs que les bacheliers STI2D qui ont pourtant suivi l'option SIN. On constate également qu'en règle générale, les étudiants de la série $\mathrm{S}$ fournissent un travail personnel plus important que ceux de la série STI2D. Le module de SIN dispensé à l'IUT de Haguenau a donc été repensé pour amener l'étudiant à mieux s'impliquer dans sa formation et à fournir un travail personnel régulier et plus important. L'apprentissage par projet expérimenté dans le cadre de ce module est une piste très intéressante pour atteindre ces objectifs. Ce papier relate cette expérience. Après un rappel des principes de l'apprentissage par projet, l'organisation du groupe

This is an Open Access article distributed under the terms of the Creative Commons Attribution License (http://creativecommons.org/licenses/by/4.0), which permits unrestricted use, distribution, and reproduction in any medium, provided the original work is properly cited. 
d'étudiant sera développée et le déroulement des séances sera détaillé. Enfin un bilan de l'expérience est dressé.

\section{Principe de l'apprentissage par projet}

L'apprentissage par projet (APP) est un modèle pédagogique fondé sur le concept d'enquête qui a pour but de stimuler la curiosité et par voie de conséquence l'investissement des étudiants [1]. Il consiste à soumettre un projet global à un groupe d'étudiants, généralement des équipes de 4 personnes, cf. III. Le projet est soumis sous la forme d'un cahier des charges ou d'une problématique illustrant une situation réaliste. Le caractère réaliste est primordial dans cette approche, car il rattache la matière étudiée par l'étudiant à des situations qu'il est susceptible de rencontrer en dehors du cadre des études, ce qui augmente significativement l'intérêt pour la matière. L'APP s'inscrit dans le cadre des stratégies holistiques d'enseignement qui favorisent le questionnement et l'autoapprentissage chez les étudiants tout en favorisant le développement des compétences requises pour répondre aux problématiques techniques et organisationnelles rencontrées dans la plupart des entreprises actuelles [2].

Le premier objectif de l'APP est d'amener l'étudiant à se prendre en charge à travers un travail collaboratif mené au sein de l'équipe. Ce travail collaboratif sous-entend une répartition claire des tâches (cf. III), ce qui garantit l'investissement de l'étudiant qui doit apporter sa contribution et répondre de son travail devant ses pairs. Ceci a pour effet de développer des habiletés de communication et de réflexion de niveaux supérieurs.

L'APP vise en l'occurrence à développer le sens créatif et critique de chaque étudiant. De ce fait, les sujets, qui s'inspirent de situations réelles ou réalistes, ont volontairement un degré d'information technique suffisamment vague permettant d'offrir a priori une grande latitude dans les choix techniques et technologiques. Il est à noter que dans le cadre du module SIN1, les sujets sont à ce jour tous proposés par les enseignants en charge du module. Néanmoins, selon le contexte, il peut être envisagé qu'un sujet soit lancé par les étudiants eux-mêmes, voire un organisme externe.

Le degré de liberté délibérément ouvert des sujets proposés a des implications tant sur les étudiants que sur l'organisation du module. Concernant les étudiants, il rend ceux-ci acteurs de leur formation et les incite à développer leur autonomie dans le travail et la recherche d'informations. D'un point de vue de l'organisation et de la mise en œuvre, l'APP se distingue des techniques dites «classiques » (c.-à-d. cours, TD, TP) par une refonte substantielle des pratiques pédagogiques.

En l'occurrence, concernant l'enseignant « tuteur » en charge de l'accompagnement, ses interventions sont en priorité basées sur le questionnement (on répond à la question de l'étudiant par une autre question), préalablement à l'apport de réponses directes (qui ne survient qu'en ultime recours, c'est-à-dire si l'ensemble des équipes est complètement bloqué). Cette technique de recadrage a pour but de stimuler la réflexion des étudiants. L'expérience de l'enseignant est ici fortement sollicitée, car il doit être en capacité d'orienter les étudiants vers les bonnes pratiques tout en laissant un degré de liberté suffisant pour maintenir la motivation. Le fort degré de liberté a priori des sujets est aussi un critère qui exige du tuteur qu'il ait suffisamment d'expérience dans la conduite de projet, ainsi que les compétences techniques et la culture générale nécessaires pour s'adapter aux différentes approches susceptibles d'être proposées par les étudiants (c.-à-d. on ne suit pas juste une « recette » de TP).

Concernant l'évaluation du travail des étudiants, l'APP nécessite également de trouver le bon dosage entre les exigences, les objectifs (qui doivent rester réalistes) et la motivation (cf. V). Sur ce plan, le but de l'APP est de de favoriser l'épanouissement de tous les 
étudiants quel que soit leur niveau de compétence préalable, de viser un niveau de compétences minimal réaliste et d'encourager l'approfondissement pour les étudiants les plus motivés et les plus avancés.

D'un point de vue de l'organisation, le module comporte des séances dédiées aux projets ou missions (cf. IV et exemple de mission en V). Le niveau de difficulté des missions proposées suit une progression qui vise à amener les étudiants au niveau de compétence minimale requise. Pour les réalisations pratiques, les étudiants ont accès à tout le matériel électronique de base nécessaire (alimentations de laboratoire, instruments de mesure, composants de base...) ainsi qu'aux logiciels ad hoc (Quartus, ModelSim...). En particulier, l'IUT de Haguenau a profité d'une opération « un étudiant, une carte » proposée par le CNFM [3] pour faire l'acquisition d'un nombre suffisant de cartes de développement FPGA permettant à chaque étudiant qui le souhaite d'emprunter une carte à l'année. Cette opération financée en partie par le projet ANR IDEFI-FINMINA [4] a grandement contribué à l'introduction de l'approche d'apprentissage par projet à l'IUT car les étudiants disposaient à volonté du matériel durant les phases de travail personnel.

\section{III.Organisation du groupe}

Bien que cela ne soit pas indispensable dans l'approche pédagogique de l'apprentissage par projet, les étudiants travaillent en groupe de quatre. Cette organisation en groupe très structurée permet de gagner en efficacité. En effet, chaque membre du groupe à une tâche spécifique à accomplir pour que le groupe puisse mener à bien les missions. Il sait qu'elle tâche lui incombe et qu'il faut qu'il l'exécute. Ainsi, chaque les étudiants est également responsabilisé vis-à-vis de leurs camarades. Cela instaure également un autocontrôle de l'avancement des tâches par les étudiants eux-mêmes. Les quatre rôles attribués sont : l'animateur, le scribe, le secrétaire, l'intendant (voir fig. 1).
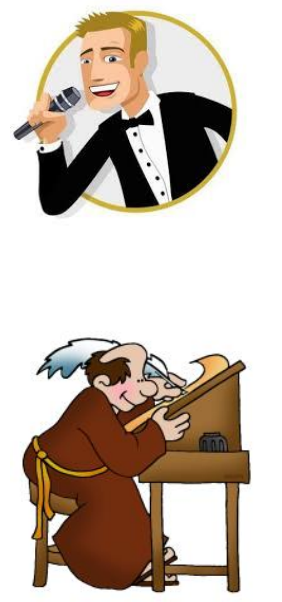

Animateur

- Discussion

- Donne la parole ou tempère

- Reformule les idées

Scribe

- Note essentielle de ce qui est dit sur un tableau

- Organise le tableau

- Pas de censure
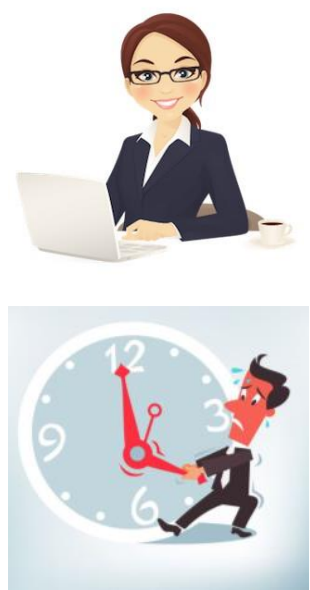

Secrétaire

- Prépare les écrits

- Synthétise les idées

- Document final

- Plan d'action

- Décisions prises

Intendant, gardien temps

- Assure le respect du temps pour les tâches

- Veille à la logistique

- Ressources matérielle

Fig. 1 Organisation du groupe. Chaque étudiant se voit attribuer un rôle bien définis pour la réalisation de la mission.

Le rôle de l'animateur est principalement d'animer la discussion. Il distribue donc la parole, suscite et sollicite la participation ou dans le cas contraire modère les interventions. À travers cette animation, il amène le groupe à clarifier les idées développées. Il peut donc être amené à proposer des synthèses de ce qui a été dit ou fait. Il veille à ce que les points importants de la discussion soient notés par le secrétaire. Finalement, il s'assure que le groupe de travail suit bien les étapes prévues. 
Le scribe est chargé de noter l'essentiel des termes, pointes, questions et idées échangés oralement. Il est le support et la mémoire de la discussion du groupe. Il est important qu'il ne filtre par les informations notées. L'accessoire idéal des scribes est un tableau dédié pour chaque groupe de projet. Le scribe a donc pour tâche d'organiser le tableau en fonction des étapes en prenant garde de laisser traces de toute la réflexion.

Le secrétaire prépare les écrites synthétiques de la production du groupe qu'il transmettra ensuite à tous les membres du groupe et au tuteur. La différence entre le secrétaire et le scribe réside, outre le support d'écriture, dans le fait que le scribe produit un document de travail en perpétuelle gestation, alors que le secrétaire synthétise un document final qui intègre notamment un plan d'action et les décisions prises par le groupe.

Le dernier rôle attribué est celui d'intendant et de gardien du temps. Il s'assure évidemment du respect du timing pour chaque étape et du timing général de la mission. Il informe donc régulièrement le groupe du temps restant pour telle ou telle étape. Il doit également veiller à la logistique en s'assurant que le groupe dispose de toutes les ressources matérielles nécessaires pour mener à bien sa mission. Cela peut aller du marqueur pour le tableau aux composants électroniques en passant par les transparents de présentation.

L'enseignant joue le rôle de tuteur dans l'approche d'apprentissage par projet. Il ne fait évidemment pas partie du groupe d'apprenants, mais il doit le guider. En effet, il est possible que les étudiants comprennent mal la mission ou bien qu'ils s'embarquent dans une solution impossible à réaliser. Le tuteur recentre donc les étudiants et les incite à aller plus loin. Il facilite aussi le travail en groupe en sollicitant les différents rôles. A priori, le tuteur n'a pas à être un spécialiste du domaine de la situation traitée, mais cela peut assurément l'aider à bien guider le groupe.

Le groupe s'organise comme il l'entend. Par conséquent, la distribution des rôles n'est pas imposée et elle n'est pas immuable. Elle peut être revue à chaque nouvelle mission. Il est même intéressant que les apprenants jouent tous les rôles au fil des séances afin de maximiser l'apprentissage.

\section{IV.Le déroulement}

La première étape de l'apprentissage passe par l'identification puis la définition des Acquis d'Apprentissage Visés (AAV). Le formateur identifie des compétences spécifiques comme par exemple, savoir mettre en œuvre un circuit intégré sur plaque labdec ou concevoir une machine à états. Une fois les AAV posés, on propose une mission qui fera intervenir les connaissances et compétences à apprendre. Cette mission dont on donne un exemple dans la section V est volontairement vague et surtout ouverte. En effet, le sujet doit donner aux étudiants une certaine liberté d'interprétation qui se peaufinera au fur et à mesure lorsqu'ils prendront conscience des ressources disponibles, notamment le matériel disponible, mais également les cours. Dans la liste des ressources disponibles, on donne également les datasheets, les logiciels, les sites web, les mots clefs pour les recherches Internet. Vient ensuite l'enchainement des séances de travaux pratiques qui se déroulent principalement en 3 phases. La phase aller, la phase retour et la restructuration (voir fig. 2). 


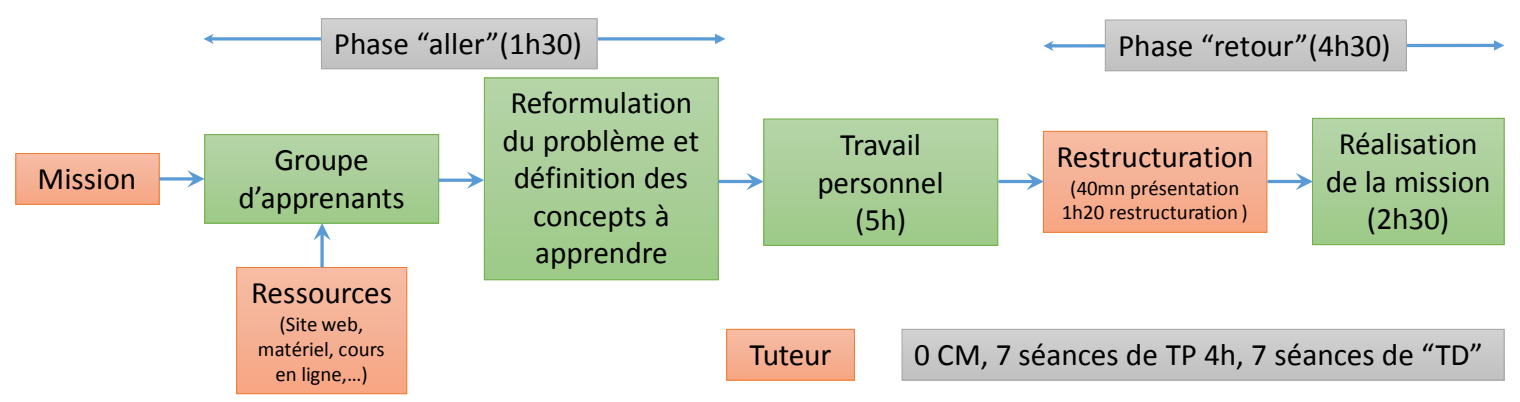

Fig. 2 Déroulement de principe des phases de l'apprentissage par projet proposé dans le cadre du modèle d'électronique numérique à l'IUT. Dans les faits, les phases aller de la mission n+1 et le réalisation de la mission $\mathrm{n}$ sont adjacent à une séance de TP. Il se passe une semaine entre deux séances de TP pour laisser le temps aux étudiants de fournir le travail personnel.

La phase « aller » : La phase aller commence toujours par l'organisation du groupe en quelques minutes (cf. III). Après l'affectation des rôles, le groupe prend connaissance du document qui comprend le sujet de la mission et la liste des ressources disponibles. La tâche suivante consiste à comprendre et reformuler le problème. Par reformulation on entend une interprétation, dans des termes techniques, d'un sujet souvent puisé dans des situations qu'ils pourraient rencontrer dans leur quotidien, afin de leur faire prendre conscience des problématiques sous-jacentes, (cf. exemple de mission à la section V). Cette tâche préliminaire est en soi déjà une phase d'apprentissage fondamentale puisqu'elle permet de développer les capacités d'abstraction, d'analyse et de hiérarchisation conceptuelle. En outre, elle pousse les étudiants à utiliser un champ lexical commun essentiel au bon déroulement de projets collectifs. Viennent ensuite les pistes pour traiter le problème, l'établissement d'une liste de questions pertinentes, et l'identification ce que le groupe connaît et surtout ce qu'il ne connaît pas. Il faut également établir une liste des livrables attendus et identifier des pistes pour avancer dans le traitement de la mission. Vient ensuite la phase essentielle des acquis d'apprentissage dans laquelle les étudiants se demandent ce qu'il faut apprendre ou découvrir pour traiter le problème. Le résultat de ces premières tâches de la phase «aller » est un plan d'action qui détermine les informations à recueillir pour confirmer ou invalider les pistes énumérées et dresse la liste des tâches à accomplir et des délivrables à préparer par chacun avant la séance « retour ». Ce plan d'action est envoyé le soir même au tuteur qui pourra ainsi juger de la pertinence du plan d'action et procéder à un recadrage si nécessaire. La durée de la phase «aller» est d'environ 1 h30 en séance. Elle est suivie d'un travail personnel de 3 à 5 heures fourni par chaque membre du groupe conformément au plan d'action. La première phase aller se déroule sur une séance dédiée de $2 \mathrm{~h}$ et les suivantes se déroulent à la fin d'une séance de $\mathrm{TP}$ de $4 \mathrm{~h}$.

La phase retour : La phase retour se déroule en deux temps. Dans un premier temps, durant une séance de 2 heures, les groupes présentent le résultat de leur travail personnel devant les autres groupes. Cette présentation d'une durée de 10 à 15 minutes reprend leur interprétation du sujet, reformule de manière synthétique et générale le problème identifié (et des problèmes secondaires s'il y a lieu), et décrit les délivrables attendus. Ils précisent ce qu'ils ont dû apprendre et les compétences qu'ils ont dû acquérir pour pouvoir mener à bien a mission. Finalement, ils présentent la solution technique qu'ils ont prévu de réaliser. Après l'ensemble des présentations qui ont duré environ une heure vient la phase de restructuration décrite dans le paragraphe suivant qui termine cette séance de 2 heures. La réalisation pratique de la solution proposée sera testée dans un deuxième temps lors d'une séance de TP de $4 \mathrm{~h}$, qui se termine par la phase «aller» de la prochaine mission comme décrit dans le paragraphe précédent. On peut également profiter de ce moment pour 
remettre un questionnaire d'autoévaluation sur les AAV. Les questions peuvent être du type : j'étais capable de construire une table de vérité avant (oui ou non) et après (oui ou non). L'étudiant peut également d'autoévaluer ces compétences finales sur une échelle de 0 à 5 qui correspond respectivement à $0:$ je ne sais rien sur le sujet, $1:$ j'ai entendu parler de ce sujet, mais je ne suis pas certain de ce que je sais, 2 :je sais dire l'une ou l'autre chose correcte à ce sujet, 3 : je sais en décrire les principaux éléments, $4:$ je sais en décrire tous les éléments pertinents ainsi que les relations entre eux, $5:$ je suis capable d'argumenter, de justifier mes affirmations, mes choix, mes décisions. Enfin, on lui demande d'évaluer le nombre d'heures qu'il a consacrées au travail personnel entre les deux séances.

La restructuration : La restructuration est intercalée dans la phase «retour» immédiatement après les présentations des solutions par les groupes durant la séance de TD. Durant cette restructuration, le tuteur montre en une heure environ comment on résout le type de problème posé en prenant soin de ne pas prendre l'exemple de la mission, mais un autre plus général. Cette phase est importante, car c'est à cette occasion que les étudiants peuvent avoir une vision claire synthétique et structurée d'une démarche de résolution d'un problème similaire à celui qu'ils ont résolu par eux même.

\section{Exemple de mission}

L'exemple de mission proposé dans cet article survient à la deuxième séance d'APP du module SIN1 au $1^{\mathrm{er}}$ semestre de $1^{\mathrm{re}}$ année GEII, vers la fin du mois de septembre ou début octobre. Ses objectifs sont multiples. Il s'agit d'une part de mettre en application des fondements de base de l'électronique numérique combinatoire (multiplexeur, démultiplexeur et encodeur de priorités). D'autre part, il s'agit de découvrir et de prendre en main un composant de type FPGA, couplé au logiciel de développement ad hoc, en l'occurrence Quartus (IntelFPGA). Les étudiants doivent donc relever plusieurs défis, ce qui rajoute à la difficulté de l'exercice. Aussi, afin de prévenir l'éventuelle accumulation d'écueils, les étudiants suivent préalablement un tutoriel d'une durée d'environ 30 à 45 minutes pour la prise en main du logiciel Quartus (création de projets, saisie d'un schéma logique, compilation, simulation et implantation de la cible FPGA).

Ils passent ensuite à la réalisation pratique de la mission qui s'intitule «Pagaille en gare! ", dont l'énoncé figure dans l'encadré ci-dessous, et pour laquelle ils ont préalablement effectué l'étude préparatoire.

\section{Mission : «Pagaille en gare !»}

Suite à un incident technique sur un tronçon des lignes régionales, tous les TER sont bloqués en gare. Seule la ligne TGV est accessible. En attendant le retour à une situation normale, il est nécessaire de trouver une solution de secours afin de rétablir le trafic (il ne s'agirait tout de même pas de rater des cours à l'IUT alors que l'année s'annonce passionnante...!). L'idée est de mettre à disposition des trains régionaux un tronçon de la ligne TGV. Le hic, c'est que le poste d'aiguillage est loin des quais et il faudrait donc un système capable de vérifier la présence ou non d'un train sur chaque quai pour permettre la régulation du trafic. Il y a en tout 7 quais. Vous êtes donc chargés de concevoir un prototype permettant de réaliser ce test.

Bon, vérifier la présence d'un train, c'est bien beau, mais ce qui serait encore mieux, ce serait de pouvoir informer les conducteurs de train qu'ils ont feu vert pour accéder au tronçon TGV. Par souci de pragmatisme, la direction des chemins de fer a décidé d'accorder l'accès dans l'ordre des quais. Ainsi, s'il y a un train sur le quai numéro 1, celui-ci a la priorité sur le quai numéro 2 et ainsi de suite. Attention néanmoins, les quais 1,2, 4 et 6 sont réservés aux TER alors que les quais 3,5 et 7 sont réservés aux TGV. Or, les TGV ont priorité sur les TER pour accéder à la ligne TGV. 
Dans un deuxième temps, vous allez donc concevoir un prototype de ce système de gestion des feux de circulation.

Vous vous dites que proposer une solution avec des circuits «74HCT-quelque-chose » a probablement ses limites. Et puis au détour d'un chemin lors d'une promenade dans les méandres des dossiers mis à votre disposition sur l'intranet par vos professeurs il vous semble avoir vu une documentation au sujet d'une carte de développement DE10-Lite comportant un circuit FPGA. Un autre document parle aussi d'un logiciel appelé «Quartus ». Vous décidez de vous y plonger coûte que coûte, et vous vous promettez d'en faire une fiche synthétique.

Au fait, avant (ça veut dire quoi «avant» d'ailleurs ?!) on appelait les conducteurs de train les chauffeurs. Savez-vous pourquoi ?

Le sujet comporte trois points importants. D'une part, les deux premiers paragraphes énoncent le cahier des charges des fonctions à réaliser. D'autre part, le troisième paragraphe suggère fortement la découverte et l'utilisation d'outils adaptés au développement rapide de fonctions complexes.

En abordant la problématique de l'aiguillage, le premier paragraphe fait une analogie directe avec le concept de multiplexeur. Quant au deuxième paragraphe, il est dédié au cahier des charges d'un encodeur de priorités.

Pour cette mission, le rôle de l'enseignant «tuteur » consiste à amener les étudiants à prendre conscience de l'importance de la hiérarchisation. Le système doit être découpé en sous-ensembles (multiplexeur, encodeur), plus facile à appréhender et à concevoir individuellement. L'enseignant insiste également sur l'intérêt et l'importance de la simulation dans la démarche de conception des systèmes électroniques modernes.

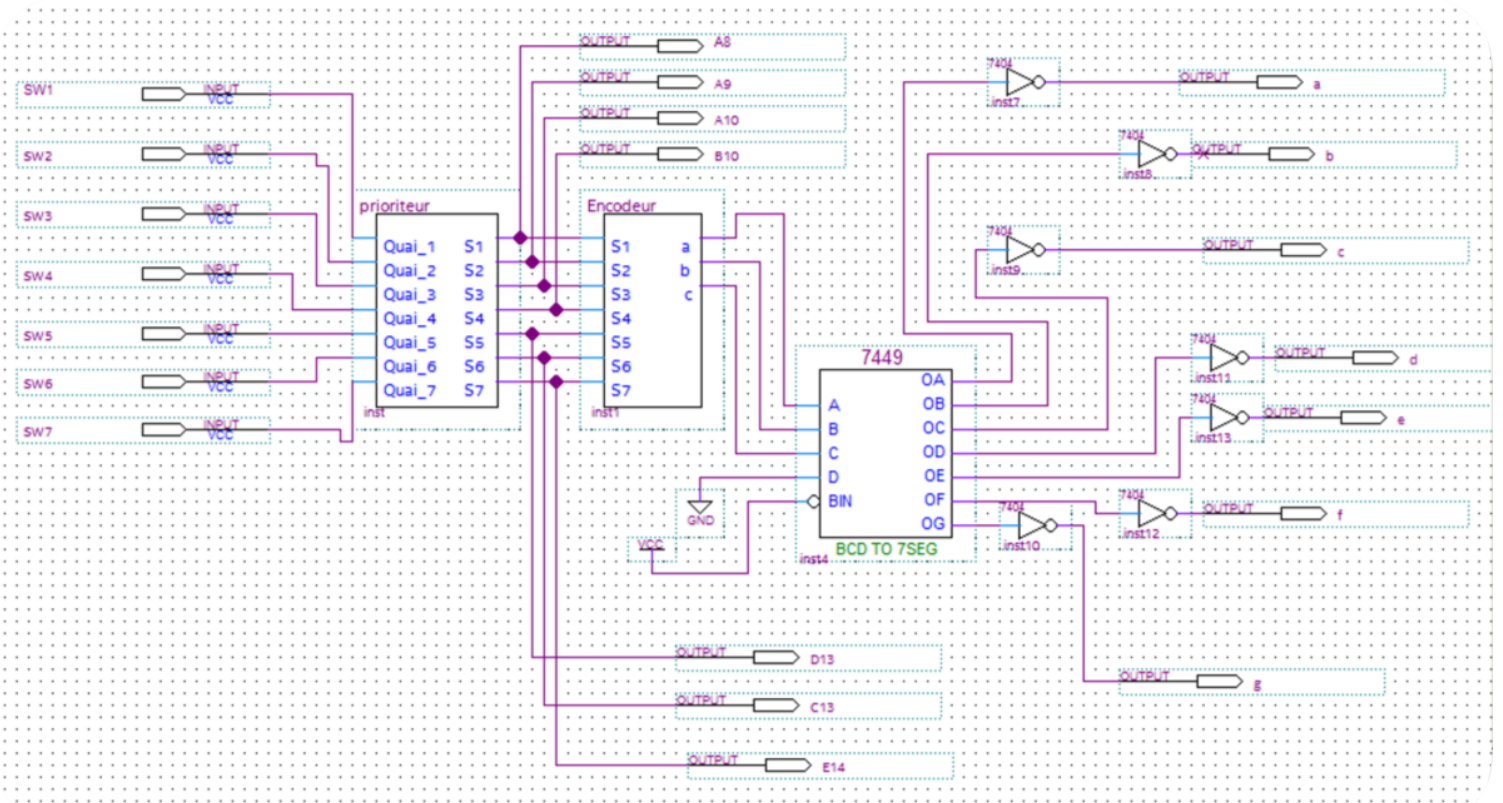

Fig. 3 Réalisation d'une solution à la mission précédente proposée par un groupe d'étudiant. Le prioriteur et l'encodeur font partie de la solution demandée. La partie transcodeur binaire codé décimale vers 7 segment est une partie rajoutée spontanément par les étudiants.

\section{Retour d'expérience}

L'approche d'APP a été très bien perçue par les étudiants sur les premières missions. La motivation et l'implication étaient au rendez-vous. Nous avons également constaté que les étudiants font preuve d'ingéniosité et parfois d'une grande originalité, en particulier dans la manière d'analyser un sujet qu'ils découvrent. Même si cette analyse est parfois 
maladroite et naïve du fait de l'inexpérience, elle apporte un souffle frais dans l'approche de la matière qui peut être profitable à la fois aux apprenants mais également aux enseignants tuteurs. En effet, à plusieurs reprises, des groupes d'étudiants ont proposé des solutions techniques en ne comprenant pas pourquoi ils n'arrivaient pas à les mettre en œuvre en pratique. L'analyse des ces propositions révélaient souvent, voire systématiquement, que même si elles semblaient viables sur le plan conceptuel ou algorithmique, la manière de les implémenter était maladroite ou erronée. La gestion des processus et des listes de sensibilité des signaux de machines d'état en langage VHDL est un exemple typique des maladresses rencontrées. Ces exemples d'erreurs sont autant de ressources qui peuvent être exploitées par l'enseignant pour illustrer les travers et écueils récurrents dans l'apprentissage d'une matière. Il est important cependant d'agir subtilement et de s'assurer que cette exploitation ne se fasse pas au détriment des étudiants qui ont fait l'erreur. Aussi, afin qu'ils ne se sentent pas stigmatisés et d'éviter le découragement, il convient d'abord de saluer l'effort et de valoriser le travail en insistant sur le fait que l'approche conceptuelles est bonne et surtout que l'erreur n'était pas évidente à voir car elle nécessite un certain degré d'expérience préalable.

Toutefois, il est reconnu que l'APP n'est pas forcément une technique d'apprentissage plus efficace que les méthodes d'enseignement classique, notamment en ce qui concerne le critère du nombre d'heures consacrées à l'apprentissage [5]. Ainsi, nous avons constaté une détérioration de l'implication des étudiants lorsque la difficulté conceptuelle s'est accentuée, notamment lors de l'introduction de la logique séquentielle. Certains étudiants semblaient submergés par le flux d'information et ont émis le besoin d'avoir des cours intégrés. Aussi, il a été constaté qu'en avançant dans le module, la spontanéité avait tendance à laisser place à une certaine uniformisation des travaux et un formalisme qui s'expliquent d'une part par l'acquisition progressive des techniques de conception et des méthodes de travail et d'autre part par une augmentation des échanges de solutions entre les groupes d'étudiants. Un autre travers souvent constaté est la récurrence des rôles joués par les étudiants. En effet, en dépit des encouragements à opérer une rotation des rôles, ils identifient très vite les compétences de chacun au sein du groupe, voire même de la promotion. Aussi, les étudiants, notamment les plus compétents techniquement sont systématiquement sollicités, voire s'imposent d'eux-mêmes, pour mener le groupe et proposer des solutions aux problématiques posées. En outre, la composition des groupes est souvent figée dès la deuxième séance et l'on constate que les étudiants éprouvant des difficultés ont tendance à se reposer sur le reste du groupe, ce qui tend à les stigmatiser et à accentuer leur retard. Ainsi des pistes d'amélioration doivent être trouvées. On pourrait par exemple imposer une recomposition systématique des groupes, ce qui favoriserait le maintien de la spontanéité et de l'originalité des propositions et inciterait les étudiants à renouveler en permanence leur engagement.

Enfin, avec l'augmentation croissante du niveau de difficulté technique, on constate que les étudiants négligent les phases de réflexion au profit de méthodes de travail «à tâtons » peu efficaces. Obnubilés par le résultat final et le désire d'aboutir vite à la phase pratique, ils tentent souvent de mettre en œuvre des fonctions toutes faites, trouvées par exemple sur internet, perdant ainsi plus de temps à les adapter au cahier des charges que s'ils avaient conçu et simulé leur propre architecture.

\section{Conclusion}

Cette expérience de l'approche d'APP été très intéressante. Les étudiants apprécient la liberté offerte par les sujets des missions. Toutefois, la maturité des étudiants de première année ne semble pas assez importante pour leur laisser la tâche d'identifier ce qu'ils ont à 
découvrir et à apprendre. C'est pourquoi, nous avons convenu que nous ne gardons cette approche que dans les cadres des TP de SIN1 et que des cours et TD associés ont été réintroduits dans le semestre 1. Nous envisageons cependant de retenter l'approche dans le cadre du module portant sur les microprocesseurs au deuxième semestre.

\section{Remerciements}

Nous remercions Denis Pénard, Gilles Raynaud et Jacques-Olivier Klein pour leur intervention sur l'apprentissage par projet lors du $44^{\mathrm{e}}$ colloque pédagogique national des départements GEII qui s'est déroulé à Calais, du 31 mai au 2 juin 2017. Nous les remercions également pour le partage de leurs supports pédagogiques qui ont servi de base de travail pour l'introduction de cette approche pédagogique à l'IUT de Haguenau. Ces actions sont partiellement soutenues grâce aux outils mis à la disposition de l'Université dans le cadre du réseau GIP-CNFM et le projet IDEFI-FINMINA.

\section{Références}

1. Joseph S. Krajcik, The Cambridge Handbook of the Learning Sciences, chapter 19 - Project-Based Learning, pp 317-334, https://doi.org/10.1017/CBO9780511816833.020

2. Philippe Perrenoud, Apprendre à l'école à travers des projets : pourquoi ? comment ?, http://www.unige.ch/fapse/SSE/teachers/perrenoud/php_main/php_1999/1999_17.html (Accès février 2019)

3. GIP-CNFM: Groupement d'Intérêt Public - Coordination Nationale pour la formation en Microélectronique et en nanotechnologies. Website: http://wwww.cnfm.fr (Accès juillet 2018)

4. IDEFI-FINMINA : Initiative d'Excellence - Formation Innovante en MIcroélectronique et Nanotechnologies, ANR-11-IDFI-0017. Website: http://www.cnfm.fr/VersionFrancaise/actualites/ FINMINA.htm

5. Jean Proulx, L'apprentissage par projet, Presses de l'Université du Québec, ISBN: 978-2760512948 